Trauma Berufskrankh $2004 \cdot 6$ [Suppl 4] : S384-S392 DOI 10.1007/s10039-003-0804-2

Online publiziert: 14. Januar 2004 (c) Springer-Verlag 2004
S. Rammelt $\cdot$ R. Grass $\cdot$ A. Biewener $\cdot$ H.Zwipp

Klinik und Poliklinik für Unfall- und Wiederherstellungschirurgie,

Universitätsklinikum „Carl Gustav Carus" der TU Dresden, Dresden

\title{
Anatomie, Biomechanik und Klassifikation der Sprunggelenkfrakturen
}

as obere Sprunggelenk (Articulatio talocruralis) ist eine anatomische Einheit aus den gelenktragenden Anteilen der distalen Tibia und Fibula, des Corpus tali sowie den verbindenden ligamentären Strukturen. Während es klassischer Weise als Scharniergelenk betrachtet wird, haben kinematische Untersuchungen gezeigt, dass die typischen Bewegungen des Talus in der Sagittalebene (ca. $50^{\circ}$ Plantarflektion und $20^{\circ}$ Dorsalflektion) von gekoppelten Bewegungen in der Frontal- und Horizontalebene begleitet sind $[15,20,25]$. Diese sind jedoch von geringem Umfang und werden in der Literatur unterschiedlich angegeben. Sasse et al. [40] wiesen eine Außenrotation des Talus von $4,2^{\circ}$ und eine Innenrotation von $1,4^{\circ} \mathrm{im}$ sagittalen Bewegungsumfang von $20 / 0 / 35^{\circ}$ nach. Michelson et al. [25] fanden geringere Werte für die Rotation und zusätzlich weniger als $1^{\circ}$ Varus-/Valgus-Bewegungen in der Horizontalebene.

Durch die ungleichförmige Form der Talusrolle (• Abb. 1) und die elastische Aufhängung der Malleolengabel vollführt die Fibula darüber hinaus bei allen Bewegungen des Talus eine dreidimensionale Relativbewegung zur Tibia $[15,30]$. Bei maximaler Dorsalflektion rotiert die Fibula um ca. $2^{\circ}$ nach außen und beschreibt eine vertikale sowie sagittale Mitbewegung nach hinten und unten $[30,39]$. Knöcherne Leitstrukturen und Bandapparat sind gleichermaßen bedeutsam für die Stabilität ( Abb. 2; $[33,53])$ im obe- ren Sprunggelenk. Neuere biomechanische Studien, welche die Aktivierung motorischer Einheiten simulierten, haben zudem die Bedeutung der muskulären Stabilisierung des oberen Sprunggelenkes während des Abrollens unterstrichen [26].

Oberes und unteres Sprunggelenk interagieren eng miteinander und bilden eine Art Kardangelenk, welches die drei- dimensionale „Maulschellenbewegung“ (Inversion) des Rückfußes, eine Kombination aus Supination, Plantarflektion und Adduktion und die entsprechenden entgegengesetzten Bewegungen bei der Eversion ermöglicht [15,51]. Beide Gelenke sind Teil einer kinetischen Kette, welche sich über das gegenläufig rotierende ChopartGelenk auf den Mittelfuß fortsetzt. Die

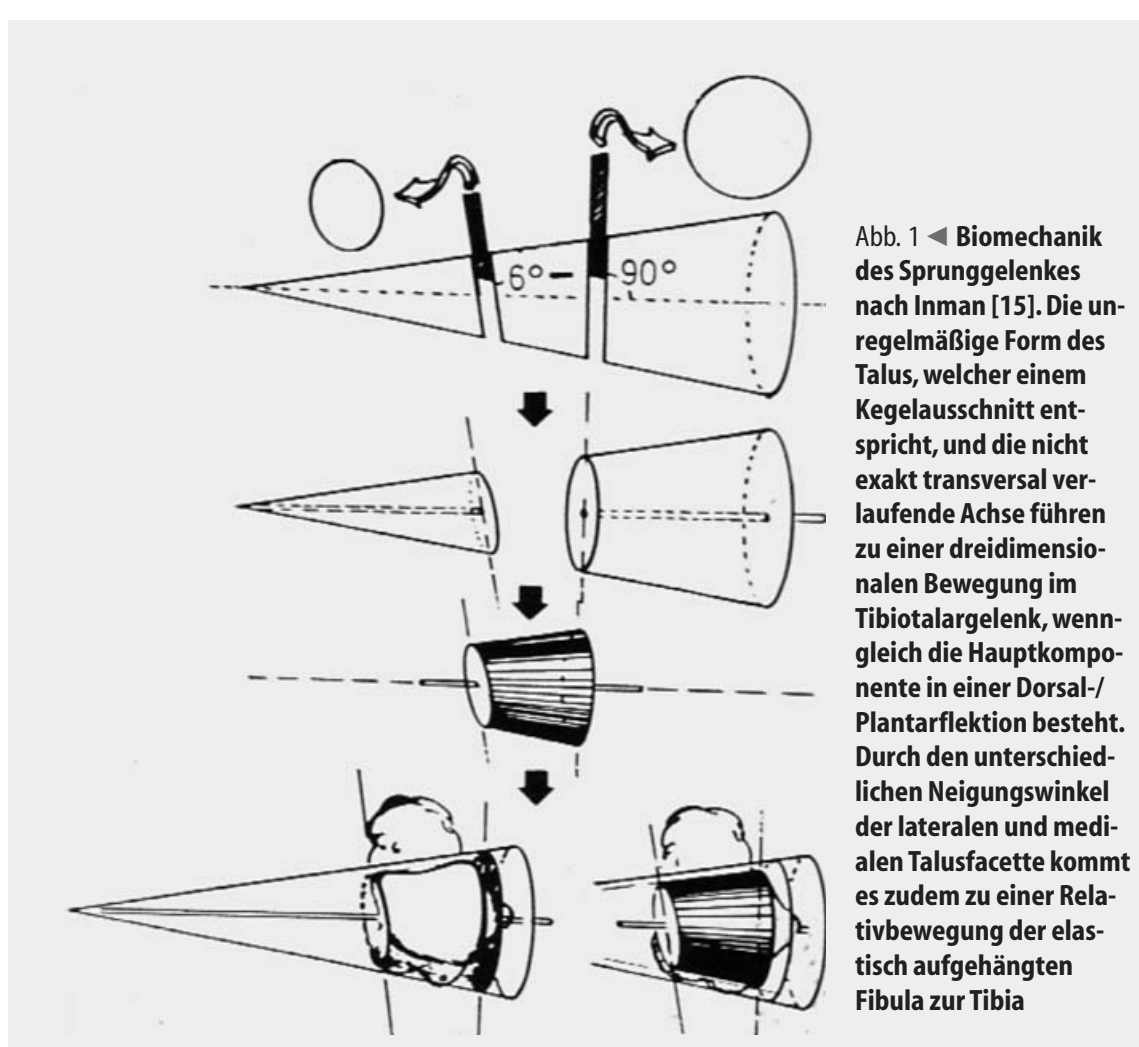


folgenden anatomischen und biomechanischen Betrachtungen sollen sich jedoch auf das obere Sprunggelenk beschränken.

\section{Anatomie}

Die distale Tibiametaphyse bildet einen kräftigen, lasttragenden Pfeiler, der von Destot [8] als „pilon“ (Stößel) bezeichnet wird. Während der Tibiaschaft einen dreieckigen Querschnitt aufweist, hat die distale Gelenkfläche die Form eines unregelmäßigen Viereckes, welches vorne breiter als hinten und lateral tiefer als medial ist. Sie begrenzt das mediane Gelenkkompartiment und bildet gleichsam das Dach („plafond“) des oberen Sprunggelenkes, welches um $15-20^{\circ}$ gegenüber der Horizontalen nach dorsal geneigt ist. Der Innenknöchel (Malleolus medialis) begrenzt das mediale Gelenkkompartiment und läuft nach distal in leichter Varusposition aus. Er besteht aus 2 kräftigen Portionen, dem Colliculus anterior und posterior, an denen die Pars anterior und posterior der tiefen Portion des Ligamentum deltoideum ansetzen, mit der gelenknahen interkollikulären Grube. Hinter dem Colliculus posterior verläuft eine Grube für die Sehnen des M. tibialis posterior und M. flexor digitorum longus.

Die distale Fibula verbreitert sich zum Außenknöchel (Malleolus lateralis) und begrenzt das laterale Gelenkkompartiment. Die hintere Knöchelspitze reicht etwa $1 \mathrm{~cm}$ weiter nach distal als der Innenknöchel. Die breite Rückfläche weist eine Rinne als Gleitlager für die Peronealsehnen auf. Die knöchernen Vorsprünge, an welchen vorderes und hinteres Syndesmosenband ansetzen (Tubercula anterius et posterius) sind etwas schwächer ausgebildet als die korrespondierenden Tubercula der lateralen Tibiametaphyse. Zwischen Letzteren befindet sich die unterschiedlich tief ausgeprägte Incisura fibularis, in welche sich die mediale Konvexizität der Fibula exakt einpasst. Die korrekte Lage der Knochen zueinander auf dieser Höhe ist von größter Bedeutung bei traumatischer Sprengung der Knöchelgabel, da hier das biomechanisch bedeutsame Ligamentum tibiofibulare interosseum mit seinen kurzen, kräftigen $\mathrm{Fa}$ -

Trauma Berufskrankh $2004 \cdot 6$ [Suppl 4] : S384-S392

DOl 10.1007/s10039-003-0804-2

C) Springer-Verlag 2004

S. Rammelt $\cdot$ R. Grass $\cdot$ A. Biewener $\cdot$ H.Zwipp

\section{Anatomie, Biomechanik und Klassifikation der Sprunggelenkfrakturen}

\section{Zusammenfassung}

Die funktionelle Integrität des oberen Sprunggelenkes wird durch eine Kombination von Formschluss, Bandführung und muskulärer Stabilisierung erreicht. Die Verletzungsanalyse beinhaltet dementsprechend neben der Frakturtopographie und -morphologie das Vorliegen von Rupturen bzw. knöchernen Avulsionen des medialen und lateralen Kollateralbandapparates sowie des tibiofibularen Syndesmosenkomplexes - zu welchem auch die Membrana interossea und die proximale Syndesmose gehören. Wichtige Kriterien für die Stabilität im oberen Sprunggelenk und damit für die Indikationsstellung zur Operation sind Länge und Stellung der distalen Fibula, die Kompetenz der medialen Strukturen und des Syndesmosenkomplexes. Die genetische LaugeHansen-Klassifikation erlaubt das Verständnis des Luxationsmechanismus und der geschlosse- nen Reposition sowie die Indikationsstellung und Planung des operativen Vorgehens in über 95\% der Sprunggelenkfrakturen. Die Danis-WeberKlassifikation muss um die Untergruppen der A0-Klassifikation ergänzt werden, um eindeutige Aussagen bezüglich der Stabilität machen zu erlauben. Kindliche Frakturen werden in Analogie zu anderen Gelenkfrakturen nach ihrer Beziehung zur Wachstumsfuge klassifiziert, wobei die Übergangsfrakturen der distalen Tibia beim 1214 jährigen Jugendlichen Sonderfälle darstellen, welcher der dreidimensionalen Analyse bedarf.

\section{Schlüsselwörter}

Oberes Sprunggelenk · Anatomie .

Pathomechanik $\cdot$ Syndesmose $\cdot$ Medialer

Bandapparat

\section{Anatomy, pathomechanics, and classification of ankle fractures}

\section{Abstract}

Functional integrity of the ankle joint is guaranteed by the shape of the joint surfaces, the capsuloligamentous attachments, and the muscles crossing the ankle. Assessment of ankle joint injuries therefore must focus not only on the fracture site and morphology but also on ligamentous ruptures or bony avulsions of the collateral and syndesmotic ligaments. Important criteria for stability and therefore indication for surgery are fibular position and shortening, competence of the medial structures, and the tibiofibular syndesmosis. The genetic LaugeHansen classification system facilitates understanding the mechanism of injury and closed reduction as well as determining stability and indications for surgery in more than $95 \%$ of ankle fractures. The Danis-Weber classification must be supplemented by the $\mathrm{AO}$ classification subgroups in order to allow assessment of the stability. Ankle fractures in children are classified according to their position with regard to the epiphyseal plate, except transitional fractures in adolescents that warrant three-dimensional assessment.

\section{Keywords}

Ankle fractures/dislocations · Anatomy . Pathomechanics - Tibiofibular syndesmosis . Medial ankle ligaments 


\section{Sprunggelenkfrakturen}
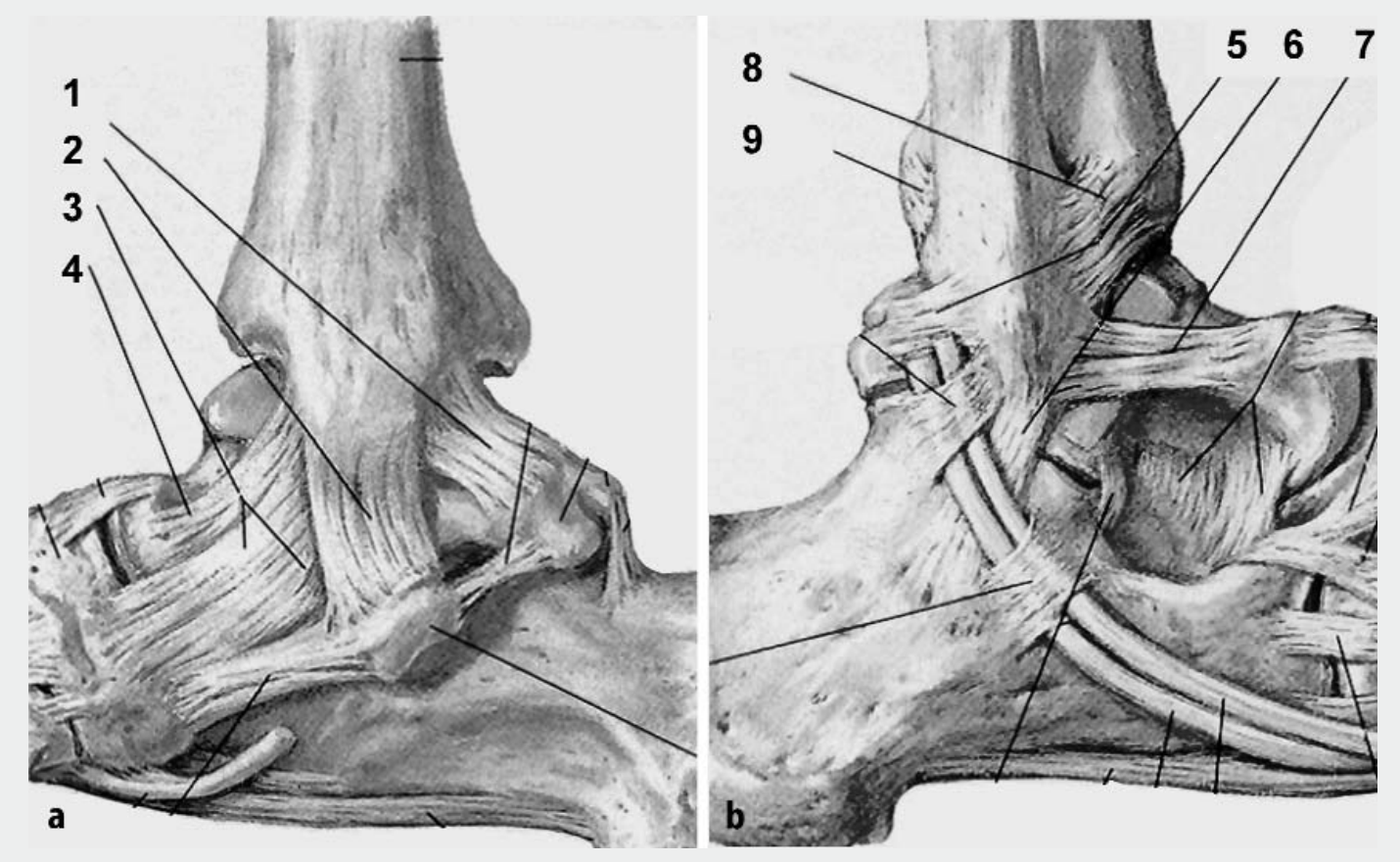

Abb. $2<$ Medialer Kollateralbandapparat (a) mit Lig. tibiotalare posterius (1), Lig. tibiocalcaneare (2), Lig. tibionaviculare (3) und Lig. tibiotalare anterius (4). Lateraler Kollateralbandapparat (b) mit Lig. fibulotalare posterius (5), Lig. fibulocalcaneare (6) und Lig. fibulotalare anterius (7). In dieser Abbildung sind auch die Ligg. tibiofibulare anterius (8) und posterius (9) erkennbar, während das kräftige Lig. tibiofibulare interosseum nur bei tiefer Präparation zur Darstellung kommt

sern die distale Fibula gegen die Tibia stabilisiert [10, 53].

Die anatomisch korrespondierende Gelenkfläche zu distaler Tibia und Fibula wird in allen 3 Kompartimenten vom Taluskörper gebildet, der aufgrund seiner Form auch als Trochlea tali (engl. „talar dome") bezeichnet wird. Nach Inman [15] stellt diese keinen Zylinder, sondern den Ausschnitt eines Kegelmantels dar, dessen Spitze nach medial zeigt. Dabei entspricht nur die mediale Talusfacette einer Kegelfläche, die laterale hingegen einer Ellipse, wodurch die Pseudorotation des Talus bei Plantar- und Dorsalflektion erklärt wird. Die Bewegungsachse des oberen Sprunggelenkes läuft direkt unterhalb der Malleolen um $8^{\circ}$ nach medial ansteigend und um $6^{\circ}$ medial nach vorn gerichtet $[15,51]$.

Tibia und Fibula sind durch den straffen Syndesmosenkomplex verbunden, welche aus dem Ligamentum tibiofibulare anterius, posterius, interosseum, transversale (innominatum) sowie dem distalen, verstärkten Anteil der Membrana interossea, besteht. Diese bietet somit ein federndes Widerlager für die Absorption der axial eingeleiteten Kräfte [10,33].

Das obere Sprunggelenk wird durch starke Kollateralbänder gegen eine Luxation des Talus aus der Knöchelgabel ge- schützt. Der mediale Bandapparat, summarisch nach seinem oberflächlichen Aspekt als Ligamentum deltoideum bezeichnet, setzt sich aus sehr heterogenen Anteilen zusammen. Das oberflächliche Deltoid besteht aus den fächerförmig ausstrahlenden Fasern der Ligamenta tibiotalare superficiale, tibiocalcaneare und tibionaviculare. Der tiefer gelegene Anteil des Ligamentum deltoideum ist deutlich kräftiger ausgeprägt und führt als Pars anterior und Pars posterior vom Innenknöchel zum Talus. Den lateralen Kollateralbandapparat bilden, in ansteigender Stärke, das Ligamentum fibulotalare anterius, Ligamentum fibulocalcaneare und Ligamentum fibulotalare posterius. Die fibularen Kollateralbänder sind in allen 3 Orientierungen des Raumes ausgerichtet [51]. Sie sind in ihrer Gesamtheit schwächer als der Innenbandapparat und wesentlich anfälliger für Rupturen beim Vertreten des Fußes [33].

\section{Biomechanik}

Die klassischen biomechanischen Untersuchungen aus den 1960er und 1970er Jahren unterstellten dem Außenknöchel die Funktion des primären Stabilisators („Leitstab") am oberen Sprunggelenk [34, 36, 45]. Tatsächlich führt insbesondere die
Verkürzung der Fibula zu einer erheblichen Fehlbelastung des Gelenkes [6, 45, 46]. Bereits die Lateralverschiebung des Talus um $1 \mathrm{~mm}$ führt zu einer etwa 40\%igen Verringerung der tibiotalaren Kontaktfläche [34, 46].

Eine Reihe jüngerer Studien belegte jedoch die hervorragende Rolle der medialen Strukturen für die Sprunggelenkstabilität $[6,24,26,35,40]$. Diese Ergebnisse erklären die Beobachtung, dass die maßgebliche Instabilität bei Sprunggelenkfrakturen nicht in einer reinen lateralen Translation des Talus, der dem frakturierten Außenknöchel folgt, sondern vielmehr in einer anterolateralen Rotation (ggf. mit Valgusstress) besteht, welche durch den frakturierten Innenknöchel oder das rupturierte Ligamentum deltoideum möglich wird [19, 22, 24]. Dies rückt den Innenknöchel und das Ligamentum deltoideum als primäre Stabilisatoren in den Mittelpunkt des Interesses.

Die differenzierte Betrachtung zeigt, dass isolierte Rupturen des oberflächlichen Anteiles des Ligamentum deltoideum (typischer Weise Avulsionen des Colliculus anterior) noch keine Instabilität hervorrufen [25, 44]. Entsprechend ist bei der operativen Versorgung von kleineren Innenknöchelfragmenten, an welchen der oberflächliche Anteil des 
Deltabandes ansetzt, kein Stabilitätsgewinn zu verzeichnen [24, 28, 44]. Die Refixation größerer, die gesamte Breite des Innenknöchels einnehmender Frakturen („suprakollikuläre Frakturen“ nach Pankovich, [28]) hingegen stabilisiert die kräftige, tiefe Portion des Ligamentum deltoideum. Klinische Studien mit Beobachtungszeiträumen von bis zu 30 Jahren bestätigen die experimentell gewonnenen Erkenntnisse, indem sie für isolierte Fibulafrakturen sehr gute Ergebnisse bei konservativ-funktioneller Therapie nachwiesen $[1,16,37,48]$, während wiederholt gezeigt werden konnte, dass bei Bimalleolarfrakturen die operative Therapie deutlich bessere Resultate erzielen lässt [31,32, 49].

Wenngleich auch die Bedeutung des Ligamentum deltoideum für die Stabilität im oberen Sprunggelenk unumstritten ist, erscheint die operative Versorgung reiner Innenbandläsionen im Rahmen von Sprunggelenkluxationsfrakturen nach dem derzeitigen Stand nicht notwendig. Die kräftigere, tiefe Portion des Ligamentum deltoideum ist bei der typischer Weise zentral gelegenen Ruptur der direkten Naht ohnehin nur schwer zugänglich [44]. Eine prospektiv-randomisierte Studie [43] sowie verschiedene Fallserien ergaben keine Verbesserung des funktionellen Behandlungsergebnisses von instabilen Sprunggelenkfrakturen (Typ Weber B und C) durch die Exploration und Naht des rupturierten Ligamentum deltoideum. Andererseits finden sich in der Literatur [3] wie auch im eigenen Krankengut fallweise chronische mediale Bandinsuffizienzen insbesondere nach PronationsEversions-Frakturen, bis hin zur Entwicklung eines posttraumatischen Pes plano valgus bei Insuffizienz des Ligamentum tibionaviculare [11], sodass auf diesem Gebiet sicherlich noch Klärungsbedarf verbleibt. Einigkeit besteht allerdings hinsichtlich der Notwendigkeit der Versorgung von Innenknöchelfrakturen im Rahmen von Sprunggelenkfrakturen.

Unter axialer Belastung übernehmen die Gelenkflächen die Funktion der primären Stabilisatoren in der Frontalebene und erhöhen den Widerstand gegen die Talusrotation in der Horizontalebene [42]. Daraus ergibt sich mit ansteigender axialer Lastaufnahme bei allen Frakturformen eine erhebliche „Stabilisationsreserve“ gegen die Translation des Talus [26,35]. Nach Stormont et al. [42] bieten die Gelenkflächen unter Belastung eine 30\%ige Stabilität gegen Rotationskräfte sowie eine 100\%ige Stabilität gegen Varus-/ValgusDislokationen.

Von maßgeblicher Bedeutung für die Stabilität des oberen Sprunggelenkes ist die Integrität der distalen tibiofibularen Syndesmose [10]. Die isolierte Ruptur des Ligamentum tibiofibulare anterius ist biomechanisch nicht bedeutsam [39]. Erst bei Ruptur der kräftigen Ligamenta tibiofibulare posterius und interosseum resultiert eine pathologische Außenrotation des Talus, ohne dass knöcherne Verletzungen vorliegen $[39,47]$. Diese kommt unter statischen Bedingungen (bei rein axialer Lasteinleitung) nicht zum Tragen, wird jedoch unter dynamischen Bedingungen (Außenrotationsstress) symptomatisch [47]. Immerhin übernimmt die distale Fibula zwischen 10 und $17 \%$ auf den Unterschenkel der eingeleiteten Last, eine Eigenschaft, welche bei Syndesmoseninsuffizienz verloren geht [30]. Verschiedene Autoren haben im Modell einer Bimalleolarfraktur mit hoher Fibulakomponente (Danis-Weber C) eine schrittweise Zunahme der Sprunggelenkinstabilität bei sequenzieller Durchtrennung der einzelnen Bandzügel beobachtet [2, 40]. Boden et al. fanden zudem, dass bei experimenteller Deltabandruptur eine Instabilität des Talus bei Durchtrennung der Syndesmose 3-4,5 mm oberhalb des Tibiaplafonds resultiert [2].

\section{Frakturmechanismus und Klassifikation}

Malleolarfrakturen entstehen in über $80 \%$ der Fälle durch einen Luxationsmechanismus infolge eines Sturzes oder Fehltritts. Wesentlich seltener sind Dezelerationstraumata im Rahmen von Verkehrsunfällen (ca.10\%), durch direkte Gewalteinwirkung werden weniger als 5\% der Knöchelverletzungen ausgelöst [51]. Wie aus den biomechanischen Betrachtungen hervorgeht, muss die Verletzungsanalyse knöcherne und ligamentäre Läsionen gleichermaßen berücksichtigen. Die Morphologie und das Ausmaß der Verletzung werden von der Stellung des Fußes und die Richtung der einwirkenden Kraft zum Unfallzeitpunkt bestimmt. Lauge-Hansen $[18,19]$ hat aufgrund klinischer Beobachtungen und experimenteller Untersuchungen in hervorragender Weise eine Verbindung zwischen Pathomechanismus und Frakturmorphologie hergestellt. Die resultierende genetische Klassifikation ist somit sowohl für das Verständnis des Frakturmechanismus als auch für die Beurteilung der Stabilität und die unmittelbare Therapiewahl wertvoll, weswegen im Folgenden der Verletzungsmechanismus anhand der Lauge-Hansen-Klassifikation abgehandelt werden soll.

\section{Genetische Klassifikation nach Lauge-Hansen}

Die Bezeichnung der 4 Grundtypen der Sprunggelenkverletzungen setzen sich aus der Stellung des Fußes (Pro-/Supination) und der Richtung der Gewalteinwirkung auf den Talus (Abduktion/Adduktion/ Eversion) zum Unfallzeitpunkt zusammen. Der von Lauge-Hansen verwendete Begriff der „Eversion“ ist hierbei missverständlich, da er synonym für die dreidimensionale Gesamtbewegung des unteren Sprunggelenkes verwendet wird [51]. Entscheidend für das Verständnis der $\mathrm{Pa}$ thomechanik ist, dass hierunter die $\mathrm{Au}$ ßenrotation des Talus verstanden wird, weswegen im englischsprachigen Schrifttum statt „Eversion“ der Begriff „,external rotation" verwendet wird. Die relative Häufigkeit anhand größerer Fallstudien ist in Klammern angegeben $[12,19,29,51]$.

1. Supinations-Adduktions-Fraktur (5-20\%)

2. Pronations-Abduktions-Fraktur (5-21\%)

3. Supinations-Eversions-Fraktur (40-75\%)

4. Pronations-Eversions-Fraktur (7-24\%)

Die verschiedenen Verletzungen verlaufen in Stadien, welche Subgruppen der Klassifikation bilden und das Ausmaß der Instabilität genau definieren, wobei jeweils alternativ knöcherne oder ligamentäre Strukturen betroffen sein können (z. B. Malleolus medialis und/oder Ligamentum deltoideum). Diese Unterteilung 


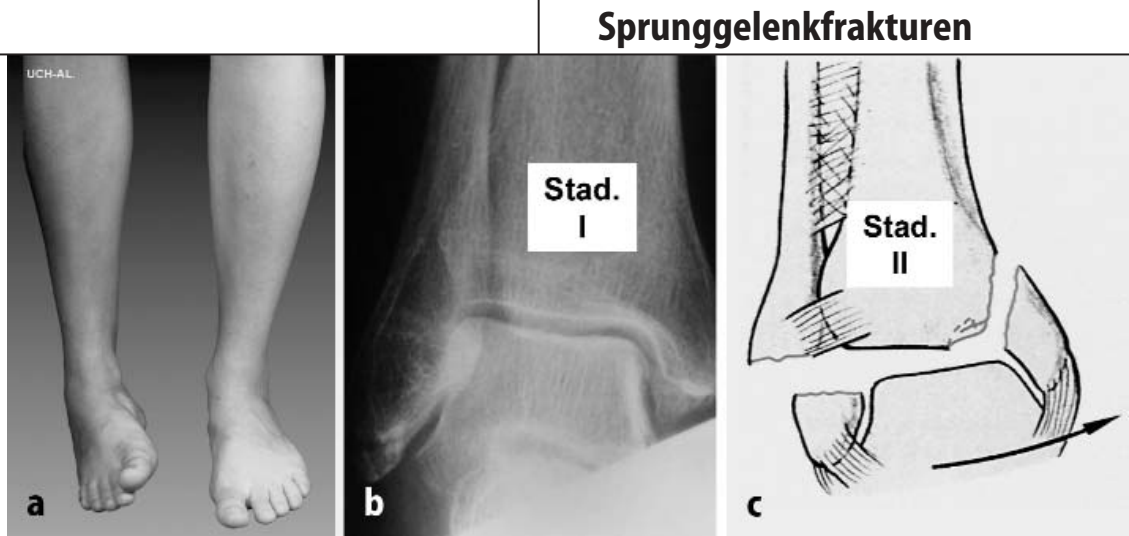

Abb. $3 \Delta$ Verletzungsmechanismus der Supinations-Adduktions-Fraktur (a) und klinisches Beispiel einer Stadium-I-Verletzung im Sinne einer distalen Bandavulsion (b). Graphische Darstellung einer Stadium-II-Verletzung mit typischer vertikal verlaufender Innenknöchelfraktur und medialer Impression des Tibiaplafonds (c)
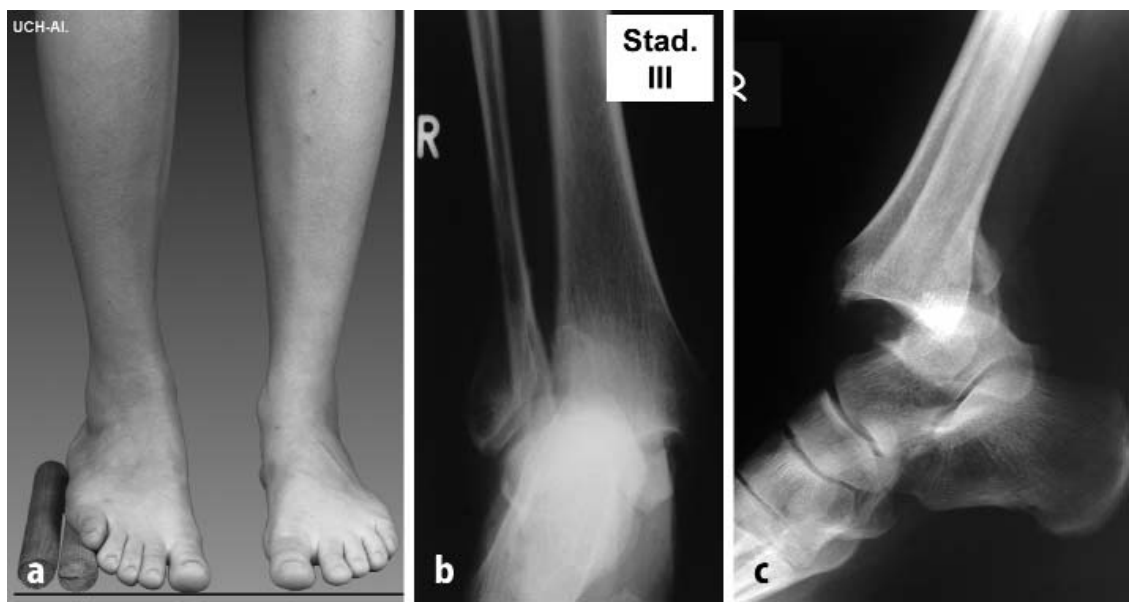

Abb. $4 \Delta$ Verletzungsmechanismus der Pronations-Abduktions-Fraktur (a) und klinisches Beispiel einer Verletzung im Stadium III mit horizontal verlaufender Innenknöchelfraktur und indirekter Trümmerfraktur der Fibula (b). Der Talus steht nach posterior subluxiert mit disloziertem knöchernen Ausriss der hinteren tibiofibularen Syndesmose (c)
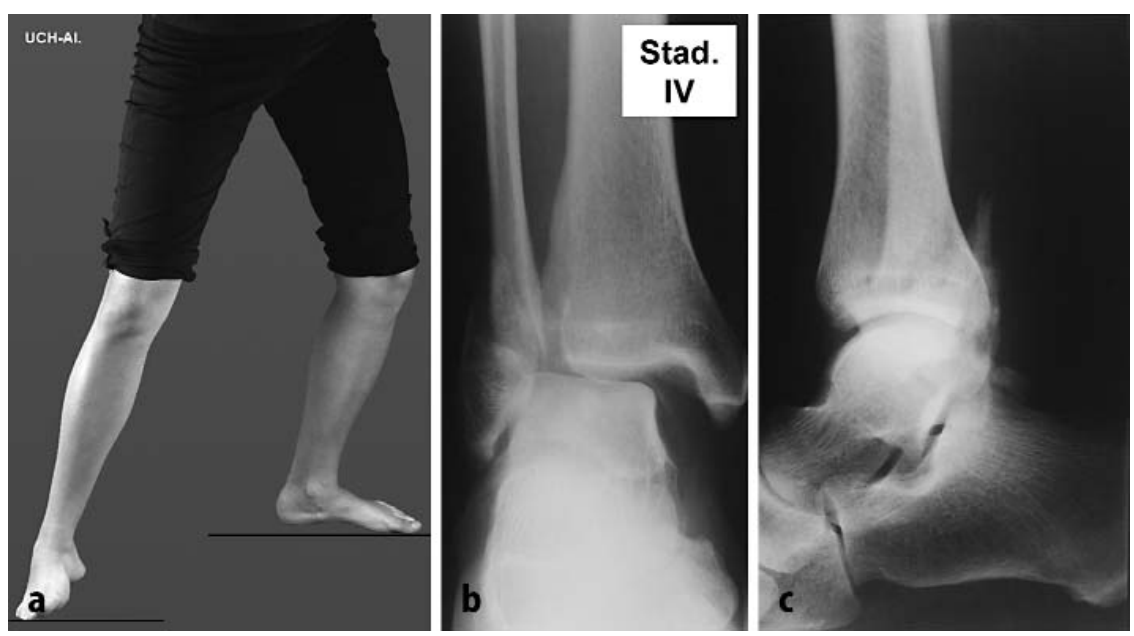

Abb. $5 \Delta$ Verletzungsmechanismus der Supinations-Eversions- (Außenrotations-)Fraktur (a) und klinisches Beispiel einer Verletzung im Stadium IV mit der typischen Torsionsfraktur der Fibula und einer Ruptur des Lig. deltoideum mit medialer Diastase (b). Schaliger knöcherner Ausriss der hinteren tibiofibularen Syndesmose im seitlichen Röntgenbild (c) macht die Lauge-Hansen-Klassifkation für den alltäglichen klinischen Gebrauch zwar deutlich „unbequemer“ als z. B. die Einteilung nach Danis und Weber [7, 45], allerdings bekommt sie dadurch - im Gegensatz zu Letzterer - erst eine prognostische und therapeutische Relevanz $[35,54]$.

\section{Supinations-Adduktions-Fraktur}

Sie entsteht durch eine axiale Gewalteinwirkung auf den supiniert stehenden Fuß (- Abb. 3), wodurch eine forcierte Adduktion des Talus resultiert. Im ersten Stadium kommt es zu einer distalen, infrasyndesmalen Fibulafraktur, ggf. mit begleitenden Verletzungen des lateralen Bandapparates. Setzt sich die adduzierende Gewalt in Richtung auf den Innenknöchel fort, entsteht eine durch die Hebelwirkung des Talus fast vertikal verlaufende Innenknöchelfraktur (Stadium II). Bei hoher Energie tritt begleitend zur Innenknöchelfraktur eine Impression des medialen Tibiaplafonds auf. Die typische Morphologie der Innenknöchelfraktur erlaubt Rückschlüsse auf den Verletzungsmechanismus auch bei rein ligamentärer Verletzung der lateralen Strukturen.

\section{Pronations-Abduktions-Fraktur}

Ihr liegt ein prinzipiell entgegengesetzter Pathomechanismus zugrunde. Die axiale Gewalteinwirkung auf den pronierten Fuß, d. h. mit erhöhter Außenkante, erzeugt eine forcierte Abduktion des Talus in der Malleolengabel (• Abb. 4).Zunächst entsteht eine horizontal verlaufende Fraktur des Innenknöchels, selten eine Deltabandruptur (Stadium I). Bei fortgesetzter Gewalteinwirkung kommt es zu einer Ruptur der hinteren und vorderen Syndesmosenbandes bzw. einem Ausriss des hinteren Volkmann-Dreieckes (Stadium II). Die proximalen Fasern des Ligamentum tibiofibulare interosseum und die distalen Anteile der Membrana interossea bleiben intakt, sodass nicht notwendigerweise eine Syndesmoseninsuffizienz resultiert. Im Stadium III schließlich entsteht eine indirekte Fibulafraktur auf Höhe der Syndesmose, welche aufgrund des Abduktionsmechanismus typischer Weise einen lateralen Biegungskeil aufweist. Regelhaft entstehen durch den indirekten Mechanismus auch Trümmerzonen der distalen 
Fibula, welche die anatomische Reposition erschweren und die Syndesmosenstabilität beeinträchtigen können, auch wenn aufgrund der Frakturhöhe keine komplette Syndesmosenruptur vorliegt. In Analogie zu den Supinations-AdduktionsFrakturen werden auch Impressionen des lateralen Tibiaplafonds beobachtet.

\section{Supinations-Eversions-Fraktur}

Diese häufige Fraktur unterscheidet sich von der Supinations-Adduktions-Fraktur dadurch, dass aufgrund der Rotation des Unterschenkels gegen den in Supinationsstellung fixierten Fuß eine Außenrotation des Talus in der Sprunggelenkgabel resultiert (• Abb. 5). Die Krafteinwirkung beginnt am vorderen Syndesmosenband, welches auch knöchern am tibialen Ansatz („Tubercule de Chaput“) oder der Fibula („Wagstaffe-/Le-Fort-Fragment“) ausreißen kann (Stadium I). Die Außenrotation des Talus führt bei fortgesetzter Gewalteinwirkung zur klassischen transsyndesmalen, schräg bis spiralförmig verlaufenden Fibulafraktur, welche auf Höhe des Tibiaplafonds beginnt und unterschiedlich weit nach proximal zieht (Stadium II).

Durch den typischen ansteigenden Frakturverlauf von anterokaudal nach posterokranial bleiben das Ligamentum tibiofibulare interosseum und posterius sowie die Membrana interossea in diesem Stadium unverletzt und die Syndesmose stabil $[35,47]$. Eine relevante Instabilität entsteht erst, wenn die zirkulär fortwirkende Gewalt zu einer Ruptur des hinteren Syndesmosenbandes (bzw. Absprengung eines hinteren Volkmann-Dreieckes) führt (Stadium III). Jedoch bleiben auch in diesem Stadium Membrana interossea und Ligamentum tibiofibulare interosseum intakt, sodass die anatomische Wiederherstellung der tibiofibulären Relation die Kompetenz des Syndesmosenkomplexes wieder herstellt. Im Stadium IV kommt es schließlich zur horizontal bis schräg verlaufenden Avulsionsfaktur des Innenknöchels bzw. Deltabandruptur.

\section{Pronations-Eversions-Fraktur}

Sie entsteht durch den prinzipiell gleichen Außenrotationsmechanismus des Talus, welcher jedoch in Pronationsstellung des Fußes in Dorsalextension erfolgt (• Abb. 6).
Der Talus stellt sich dadurch mit dem breiteren Anteil der Trochlea in der Sprunggelenkgabel ein und schlägt zunächst den Innenknöchel horizontal weg bzw. führt zu einer Deltabandruptur (Stadium I). Die fortgesetzte Außenrotation des aus seiner medialen Aufhängung befreiten und dadurch in eine Valgusfehlstellung eintretenden Talus resultiert im Stadium II in einer kompletten Ruptur des Ligamentum tibiofibulare anterius, interosseum und posterius (bzw. korrespondierenden knöchernen Avulsionen) und somit einer Syndesmoseninstabilität. Im Stadium III entsteht durch die Varusstellung des Talus und Ruptur der Syndesmose eine hohe, suprasyndesmale Fibulafraktur. Im Sonderfall der Maisonneuve-Verletzung resultiert nach kompletter Ruptur der Membrana interossea eine proximale (subkapitale) Fibulafraktur oder eine Ruptur der proximalen tibiofibularen Syndesmose mit entsprechender Luxation des Fibulaköpfchens [21].Im Stadium IV schließlich rupturiert das Ligamentum tibiofibulare transversum als letzter verbliebener Stabilisator des Talus in der Knöchelgabel.

Weniger als 5\% aller Sprunggelenkfrakturen lassen sich nicht in eine der genannten 4 Kategorien einfügen $[19,50]$. Hierzu gehören v. a.Verletzungen, welche durch ein erhebliches Stauchungsmoment ausgelöst werden, wie die isolierte Fraktur des hinteren Volkmann-Dreieckes [23] oder die Kombination aus Innenknöchelfraktur und vorderem Kantenabbruch, von Lauge-Hansen in einem Fallbericht als Pronations-Dorsiflektions-Verletzung bezeichnet [19]. Hierbei handelt es sich möglicher Weise um Übergangstypen zu Pilon-tibiale-Frakturen. Auch Frakturen, welche durch direkte Gewalteinwirkung auf die Malleolarregion entstehen, folgen nicht den genannten Luxationsmechanismen und bedürfen einer individuellen Frakturanalyse [19]. Sehr selten werden verhakte vordere oder hintere Dislokationen der Fibula gesehen, welche eine offene Reposition erfordern, wie bei der sog. Bosworth-Fraktur [14]. Die Besonderheiten kindlicher Frakturen sind unten angeführt.

\section{Danis/Weber- und A0-Klassifikation}

In seinem Standardwerk über die Verletzungen des oberen Sprunggelenkes griff Weber [45] die pathologisch-anatomische Klassifikation des belgischen Chirurgen Danis aus dem Jahre 1949 auf [7], welche sich ausschließlich auf die Frakturhöhe der Fibula in Bezug auf den Syndesmosenkomplex bezieht. Sie unterscheidet für die Luxationsfrakturen zwischen infrasyndesmaler Fibulafraktur (Typ A) mit intakter Syndesmose, transsyndesmaler Fraktur (Typ B), bei welcher der Syndesmosenkomplex meist verletzt, jedoch nicht notwendigerweise instabil ist, und den generell instabilen suprasyndesmalen Frakturen (Typ C). Diese Frakturklassifikation hat sich aufgrund ihrer Einfachheit für die alltägliche Anwendung rasch durchgesetzt, sie erlaubt jedoch keine sichere Aussage bezüglich der Stabilität und damit zur Op.-Indikation, insbesondere bei den häufigen Typ-B-Frakturen. Ebenso wenig finden Bandläsionen und Verletzungen der medialen, anterioren und posterioren Strukturen Beachtung.

Die AO-Klassifikation [27] ergänzt die von Danis und Weber vorgenommene Einteilung um nach der Schwere der Begleitverletzungen ansteigende Untergruppen (A1.1 bis C3.3]. Sie erlaubt damit insbesondere eine therapierelevante Differenzierung der Weber-B-Frakturen in stabile und instabile Frakturtypen. Während B1-Verletzungen isolierte Außenknöchelfrakturen darstellen, tritt bei B2-Verletzungen eine Fraktur des Innenknöchels hinzu, womit eine instabile Situation vorliegt. Analog kann zwischen stabilen (A1) und instabilen (A2) Typ A-Verletzungen mit Beteiligung des Innenknöchels unterschieden werden. Die Untergruppen erlauben jedoch keine eindeutigen Rückschlüsse auf den Frakturmechanismus.

Im Sprachgebrauch hat sich zudem eine rein deskriptive Einteilung nach uni-, bioder trimalleolären (letztere erstmals von Cotton 1915 [4] als „,new type fracture “ bezeichnet) Sprunggelenkfrakturen eingebürgert. Die kräftige hintere Tibiakante tritt hier gewissermaßen als „dritter Knöchel“ hinzu [13]. Das entstehende Fragment wurde erstmals im Jahre von Earle 1828 beschrieben. Im deutschen Sprach- 


\section{Sprunggelenkfrakturen}
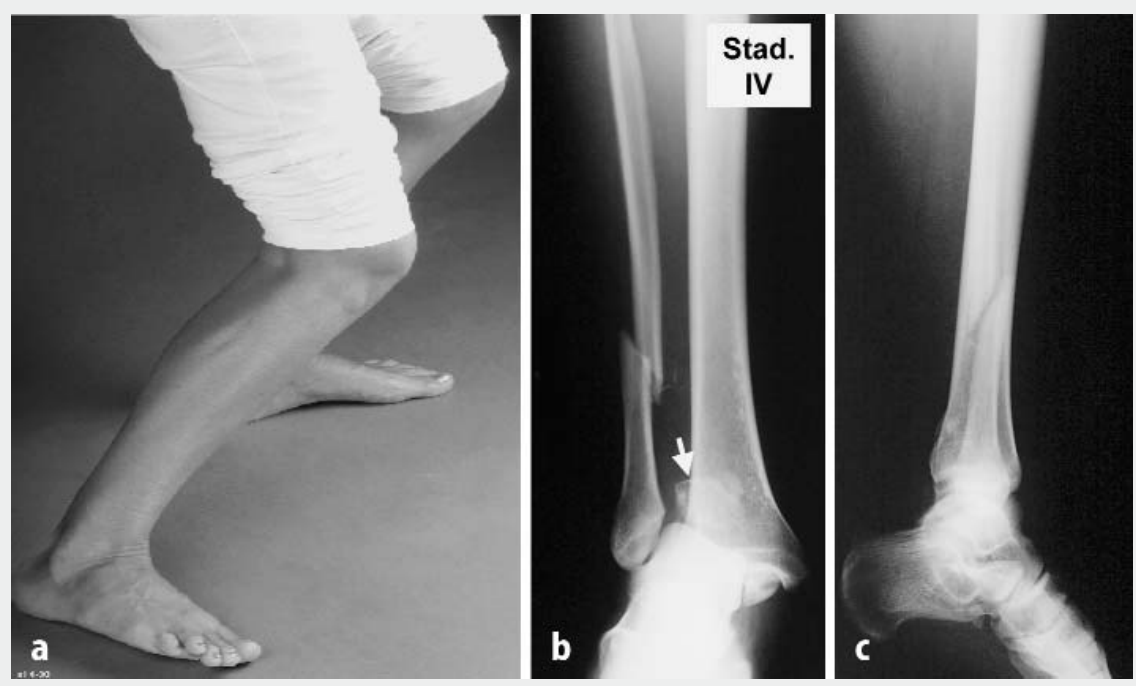

Abb. $6<$ Verletzungsmechanismus der Pronations-Eversions- (Außenrotations-)Fraktur (a) und klinisches Beispiel einer Verletzung im Stadium IV mit horizontaler Innenknöchelfraktur, disloziertem hinteren Volkmann-Dreieck (Pfeil), hoher Torsionsfraktur der Fibula und einer erheblichen tibiofibularen Diastase als Zeichen der Syndesmoseninsuffizienz $(b, c)$
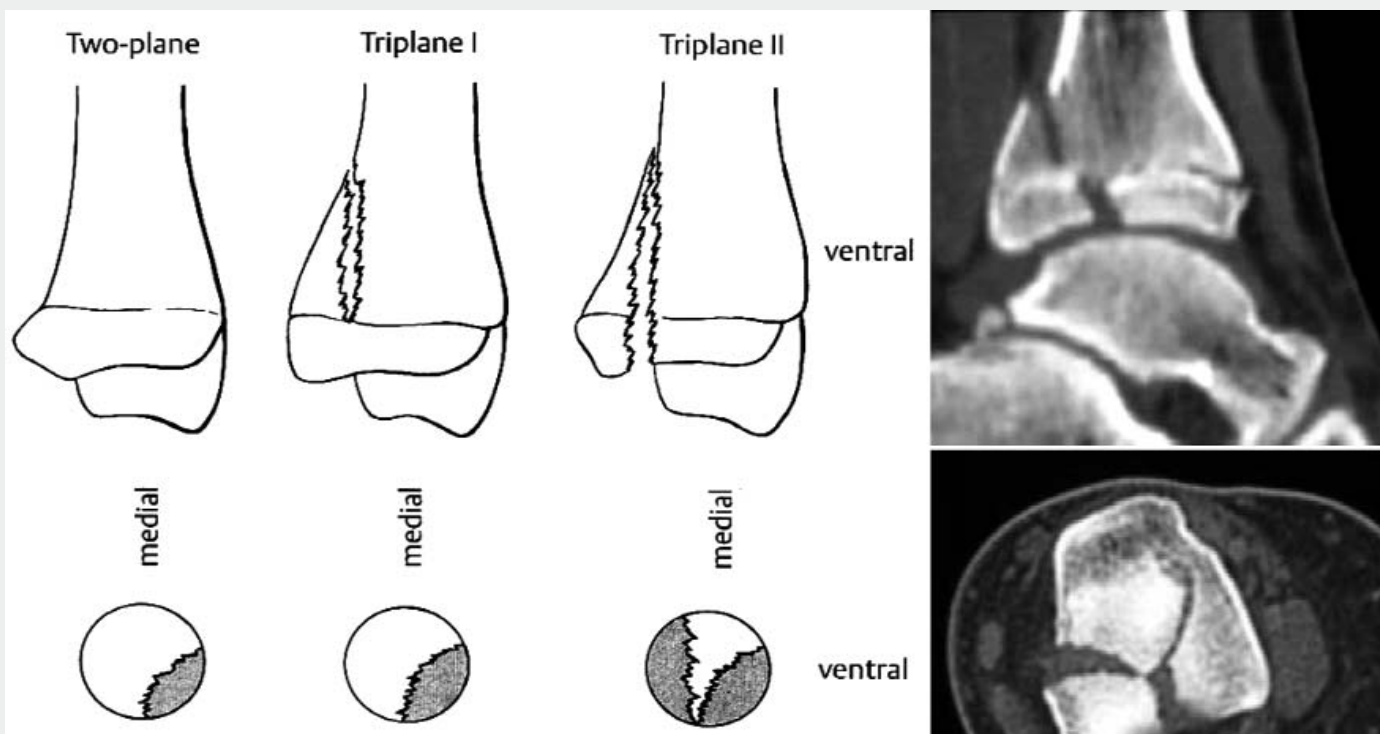

营
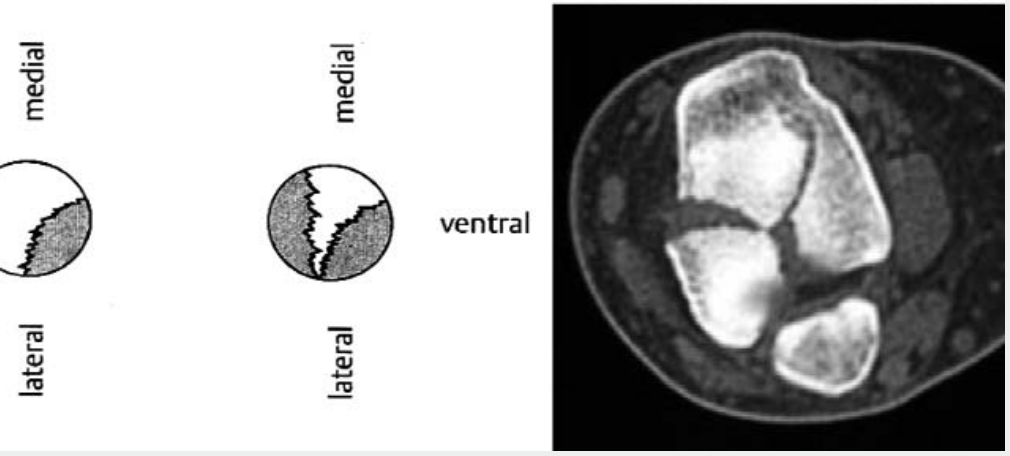

Abb. $7<$ Einteilung der Übergangsfrakturen aus dem klassischen Lehrbuch von v. Laer [17] und klinisches Beispiel einer Triplane-II-Verletzung bei einem 13-jährigen Knaben. Die Lokalisation der Frakturlinien und der Epiphysenfugenverletzung in allen 3 Ebenen wird im CT deutlich

raum wird es in Analogie zu dem von Volkmann 1875 erstmalig versorgten ventralen Kantenfragment als , hinteres Volkmann'sches Dreieck“ bezeichnet.

Ohnehin haben sich in der langen Geschichte der Frakturbehandlung am oberen Sprunggelenk zahlreiche Eigennamen für spezielle Frakturformen herausgebildet, die in unterschiedlichen Schulen bis heute in Gebrauch sind [51]. Hierzu gehören die bereits oben angeführten knöchernen Syndesmosenausrisse, wobei die Avulsionsverletzung des Ligamentum tibiofibulare anterius an der lateralen Tibia- vorderkante bereits 1822 von Cooper dargestellt und erst wesentlich später von Tillaux und Chaput in anderem Zusammenhang aufgegriffen wurde. Die von den chirurgischen „Urvätern“ Pott und Dupuytren beschriebenen hohen Fibulafrakturen werden traditionsgemäß zuweilen noch mit deren Namen bedacht [54].

\section{Klassifikation kindlicher Sprunggelenkfrakturen}

Kindliche Frakturen des oberen Sprunggelenkes werden in Analogie zu den übri- gen gelenknahen Frakturen anhand ihrer Beziehung zur epiphysären Wachstumsfuge nach Aitken bzw. Salter und Harris [38] eingeteilt, wenngleich prinzipiell die gleichen Frakturmechanismen wie von Lauge-Hansen beschrieben angenommen werden [5, 52]. Eine Sonderheit stellen die sog. Übergangsfrakturen (Biplane- und Triplane-Frakturen] der distalen Tibia dar [17]. Sie resultieren aus dem sequenziellen Epiphysenfugenschluss im Adoleszentenalter, welche Frakturlinien in bis zu 3 Ebenen, frontal, sagittal und horizontal, erkennen lassen. 
Die distale Wachstumsfuge der Tibia, welche für den Großteil des Längenwachstums des Unterschenkels verantwortlich ist, verknöchert über einen Zeitraum von etwa 18 Monaten im Alter von 12.5 bis 14 Jahren. Dieser Prozess beginnt am Übergang vom medialen zum mittleren Drittel der Epiphyse und setzt sich zunächst nach medial und dann nach lateral fort, wobei als letzter Abschnitt der vordere, laterale Epiphysenfugenanteil verknöchert. Aus diesem Grunde sind jugendliche isolierte, epiphysäre Verletzungen des „Tubercule de Tillaux-Chaput“ im Sinne einer Salter-Harris-II- bzw. „Twoplane-Fraktur" relativ häufig, während diese Verletzungen im Erwachsenenalter fast ausschließlich in der Sequenz der Malleolarfrakturen bei fortdauernder Gewalteinwirkung als zusätzliche Verletzungen auftreten.

Bildet sich ein zusätzlicher dorsaler metaphysärer Keil, so liegt eine „Triplane-Fraktur" vor [17]. Endet die metaphysäre Fraktur im Bereich der Epiphysenfuge, handelt es sich um eine „Triplane-IFraktur", setzt sie sich in die Epiphyse fort und bildet somit eine 2., dorsale Epiphysenfraktur, so spricht man von einer „Triplane-II-Fraktur“ (• Abb. 7; [17]).

\section{Fazit für die Praxis}

Die dynamische Funktion des oberen Sprunggelenkes wird durch eine Kombination von Formschluss, Bandführung und muskulärer Stabilisierung erreicht. Die Verletzungsanalyse muss daher neben der Frakturmorphologie das Vorliegen von Rupturen bzw. knöchernen Avulsionen des medialen und lateralen Kollateralbandapparates sowie des tibiofibularen Syndesmosenkomplexes - zu welchem auch die Membrana interossea und die proximale Syndesmose gehören - berücksichtigen. Entscheidend für die Stabilität im oberen Sprunggelenk und die Indikationsstellung zur Operation ist die Länge und Stellung der distalen Fibula, die Kompetenz der medialen Strukturen und des Syndesmosenkomplexes. Die genetische Lauge-Hansen-Klassifikation erlaubt das Verständnis des Luxationsmechanismus, die Indikationsstellung und Planung des operativen Vorgehens in über $95 \%$ der Sprunggelenkfrakturen. Die Danis-WeberKlassifikation muss um die Untergruppen der A0-Klassifikation ergänzt werden, um ein- deutige Aussagen bezüglich der Stabilität machen zu können. Kindliche Frakturen werden in Analogie zu anderen Gelenkfrakturen nach ihrer Beziehung zur Wachstumsfuge klassifiziert, wobei die Übergangsfrakturen der distalen Tibia beim 12- bis 14-jährigen Jugendlichen einen Sonderfall darstellen, welcher der dreidimensionalen Analyse bedarf.

\section{Korrespondierender Autor \\ Dr. S. Rammelt}

Klinik und Poliklinik für Unfallund Wiederherstellungschirurgie, Universitätsklinikum „Carl Gustav Carus“ der TU Dresden,

Fetscherstraße 74,01307 Dresden

E-mail: strammelt@hotmail.com

Interessenkonflikt: Der korrespondierende Autor versichert, dass keine Verbindungen mit einer Firma, deren Produkt in dem Artikel genannt ist, oder einer Firma, die ein Konkurrenzprodukt vertreibt, bestehen.

\section{Literatur}

1. Bauer M, Jonsson K, Nilsson B (1985) Thirty-year followup of ankle fractures. Acta Orthop Scand 56: 103-106

2. Boden SD, Labropoulos PA, McCowin P, Lstini WF, Hurwitz SR (1989) Mechanical considerations for the syndesmotic screw: A cadaver study.J Bone Joint Surg (Am) 71: 1548-1555

3. Clanton TO (1999) Athlethic injuries to the soft tissues of the foot and ankle. In:Mann RA, Coughlin MJ (eds) Surgery of the foot and ankle. Mosby, St Louis, 10901209

4. Cotton FJ (1915) A new type of ankle fracture. JAMA 64: 318-321

5. Crawford AH (1994) Fractures and dislocations of the foot and ankle. In:Green NE, Swiontkowski MF (eds) Skeletal trauma in children. Saunders, Philadelphia,

6. Curtis MJ, Michelson JD, Urquhart MW, Byank RP, Jinnah RH (1992) Tibiotalar contact and fibular malunion in ankle fractures. A cadaver study. Acta Orthop Scand 63: 326-329

7. Danis R (1949) Théorie et practique de l'ostéosynthèse. Desoer et Masson, Liège, Paris

8. Destot $E$ (1911) Traumatismes du pied et rayons $X$. Malleoles, astragale, calcaneum, avant-pied. Masson, Paris

9. Dupuytren $G$ (1847) On diseases and injuries of bones. Trans Sydenham. F le Gros Clarke, London

10. Grass R, Herzmann K, Biewener A, Zwipp H (2000) Verletzungen der unteren tibiofibularen Syndesmose. Unfallchirurg 103: 520-532

11. Grass R, Rammelt S, Biewener A, Zwipp H (2003) Aktuelle Überlegungen zur Behandlung von OSG-Frakturen. Trauma Berufskrankh 5: 141-148

12. Hamilton WC (1984) Traumatic disorders of the ankle. Springer, Berlin Heidelberg New York

13. Henderson MS (1932) Trimalleolar fracture of the ankle. Surg Clin North Am 12: 867-872

14. Hoblitzell RM, Ebraheim NA, Merritt T, Jackson WT (1990) Bosworth fracture-dislocation of the ankle. A case report and review of the literature. Clin Orthop 257-262
15. Inman VT (1976) The joints of the ankle. Williams \& Wilkins, Baltimore

16. Kristensen KD, Hansen T (1985) Closed treatment of ankle fractures. Stage II supination-eversion fractures followed for 20 years. Acta Orthop Scand 56: 107-109

17. Laer L v (2001) Frakturen und Luxationen im Wachstumsalter. Thieme, Stuttgart

18. Lauge-Hansen N (1948) Fractures of the ankle. Analytichistoric survey as the basis of new experimental, roentgenologic and clinical investigations. Arch Surg 56: 259-317

19. Lauge-Hansen N (1950) Fractures of the ankle II: Combined experimental/surgical and experimental roentgenologic investigation. Arch Surg 60: 957-985

20. Lundberg A (1989) Kinematics of the ankle and foot. In vivo roentgen stereophotogrammetry. Acta Orthop Scand Suppl 233: 1-24

21. Maisonneuve MJG (1840) Recherches sur la fracture du peroné. Arch Gén de Med 7: 165-187

22. McCullough CJ, Burge PD (1980) Rotatory stability of the load-bearing ankle. An experimental study.J Bone Joint Surg Br 62: 460-464

23. Meenen NM, Lorke DE, Westerhoff M, Dallek M, Jungbluth KH (1993) Die isolierte Fraktur des Volkmannschen Dreiecks - ein eigenständiges Verletzungsbild. Unfallchirurgie 19: 98-107

24. Michelson JD, Waldman B (1996) An axially loaded model of the ankle after pronation external rotation injury. Clin Orthop 285-293

25. Michelson JD, Checcone M, Kuhn T, Varner K (2001) Intra-articular load distribution in the human ankle joint during motion. Foot Ankle Int 22: 226-233

26. Michelson JD, Hamel AJ, Buczek FL, Sharkey NA (2002) Kinematic behavior of the ankle following malleolar fracture repair in a high-fidelity cadaver model.J Bone Joint Surg Am 84: 2029-2038

27. Müller ME, Nazarian S, Koch P (1987) Classification AO des fractures. Springer, Berlin Heidelberg New York

28. Pankovich AM, Shivaram MS (1979) Anatomical basis of variability in injuries of the medial malleolus and the deltoid ligament. II.Clinical studies. Acta Orthop Scand 50: 225-236

29. Paul D, Eckardt B, Kühnel K, Schuhmann HD (1968) Erfahrungen mit der konservativen Behandlung von Malleolarfrakturen. Arch Orthop Unfallchir 63: 273-287

30. Peter RE, Harrington RM, Henley MB, Tencer AF (1994) Biomechanical effects of internal fixation of the distal tibiofibular syndesmotic joint:comparison of two fixation techniques. J Orthop Trauma 8: 215-219

31. Pettrone FA, Gail M, Pee D, Fitzpatrick T, Van Herpe LB (1983) Quantitative criteria for prediction of the results after displaced fracture of the ankle.J Bone Joint Surg Am 65: 667-677.

32. Phillips WA, Schwartz HS, Keller CS et al. (1985) A prospective, randomized study of the management of severe ankle fractures. J Bone Joint Surg Am 67: 67-78

33. Rammelt S, Grass R, Biewener A, Zwipp H (2003) Bandverletzungen am oberen Sprunggelenk. Trauma Berufskrankh 5: 170-178

34. Ramsey PL, Hamilton W (1976) Changes in tibiotalar area of contact caused by lateral talar shift.J Bone Joint Surg Am 58: 356-357

35. Richter J, Schulze W, Clasbrummel B, Muhr G (2003) Beitrag der Syndesmose und des Deltabandes zur Stabilität der Außenknöchelfraktur Typ Weber B. Eine experimentelle Untersuchung. Unfallchirurg 106: 359-366

36. Riede U, Willenegger H, Schenk R (1969) Experimenteller Beitrag zur Erklärung der sekundären Arthrose bei Frakturen des oberen Sprunggelenkes. Helv Chir Acta 36: $343-348$ 
37. Ryd L, Bengtsson S (1992) Isolated fracture of the lateral malleolus requires no treatment. 49 prospective cases of supination-eversion type II ankle fractures. Acta Orthop Scand 63: 443-446

38. Salter RB, Harris WR (1963) Injuries involving the epiphyseal plate. J Bone Joint Surg Am 45: 587-622

39. Sarsam IM, Hughes SP (1988) The role of the anterior tibiofibular ligament in talar rotation. Injury 14: $357-$ 360

40. Sasse M, Nigg BM, Stefanyshyn DJ (1999) Tibiotalar motion - effect of fibular displacement and deltoid ligament transection: in vitro study. Foot Ankle Int 20: 733-737

41. Solari J, Benjamin J, Wilson J, Lee R, Pitt M (1991) Ankle mortise stability in Weber C fractures:indications for syndesmotic fixation.J Orthop Trauma 5: 190-195

42. Stormont DM, Morrey BF, An KN, Cass JR (1985) Stability of the loaded ankle. Relation between articular restraint and primary and secondary static restraints. Am J Sports Med 13: 295-300

43. Stromsoe K, Hoqevold HE, Skjeldal S, Alho A (1995) The repair of a ruptured deltoid ligament is not necessary in ankle fractures. J Bone Joint Surg Br 77: 920-921

44. Tornetta P, 3rd (2000) Competence of the deltoid ligament in bimalleolar ankle fractures after medial malleolar fixation. J Bone Joint Surg Am 82: 843-848

45. Weber BG (1966) Verletzungen des oberen Sprunggelenkes. Aktuelle Probleme in der Chirurgie, Bd 3. Huber, Bern

46. Weber BG (1981) Lengthening osteotomy of the fibula to correct a widened mortice of the ankle after fracture. Int Orthop 4: 289-293

47. Xenos JS, Hopkinson WJ, Mulligan ME, Olson EJ, Popovic NA (1995) The tibiofibular syndesmosis. Evaluation of the ligamentous structures, methods of fixation, and radiographic assessment. J Bone Joint Surg Am 77: 847856

48. Yde J, Kristensen KD (1980) Ankle Fractures, supinationeversion fracture stage IV, primary and late results of operative and non-operative treatment. Acta Orthop Scand 51: 981-990

49. Yde J, Kristensen KD (1980) Ankle Fractures, supinationeversion fracture stage II, primary and late results of operative and non-operative treatment. Acta Orthop Scand 51:695-702

50. Yde J (1980) The Lauge-Hansen classification of malleolar fractures. Acta Orthop Scand 51: 181-192

51. Zwipp H (1994) Chirurgie des Fußes. Springer, Wien New York

52. Zwipp H, Dahlen C, Holch M, Rammelt S (1999) Malleolarfrakturen und Frakturen des Fußskelettes. OP J 15: 160-167

53. Zwipp H, Rammelt S, Grass R (2002) Ligamentous injuries about the ankle and subtalar joints. Clin Pediatr Med Surg 19: 195-229

54. Zwipp H, Rammelt S (2002) Frakturen und Luxationen. In:Wirth CJ, Zichner L (Hrsg) Orthopädie und Orthopädische Chirurgie, Bd 8. Thieme, Stuttgart, S 531-618

\section{TraumaundBerufskrankheit.springer.de}

\section{„Online First"}

Trauma und Berufskrankheit informiert Sie regelmäßig über praxisrelevante Themen und die neuesten wissenschaftlichen Erkenntnisse Ihres Fachgebiets. Um die Publikationszeiten zu verkürzen und Ihnen einen noch schnelleren Zugriff auf aktuelle Beiträge zu ermöglichen, heißt es ab sofort "Online First".

Unter dem Begriff „Archiv" finden Sie auf der Zeitschriftenhomepage den Zugang zum elektronischen Volltextarchiv Ihrer Zeitschrift. Hier stehen Ihnen unter „Online First" aktuelle Beiträge bereits vor dem Erscheinen der gedruckten Ausgabe als HTML-Version zur Verfügung. Jeder "OnlineFirst"-Beitrag ist durch eine elektronische Identifizierungsnummer, den Digital Object Identifier (DOI), eindeutig gekennzeichnet, sofort zitierfähig und suchbar.

Als besonderen Zusatznutzen enthält die HTML-Version Referenz-Verlinkungen, die Sie direkt zu den Originalzitaten auf SpringerLink, ChemPort und Pubmed führen. Ein Service, der Ihnen ein Mehr an Informationen in Ihrem Fachgebiet sichert.

Haben Sie noch Fragen oder Anmerkungen?

Wir freuen uns über Ihre E-Mail an

springerlink@springer-sbm.com

Ihre Redaktion Fachzeitschriften Medizin/Psychologie 